\section{The Crystal Ball of Pill Bottles?}

\author{
Armin Shahrokni, MD, MPH, and Manpreet K. Boparai, PharmD, CGP, BCACP
}

$I^{n}$ $\mathrm{n}$ just 2 decades, the average number of prescribed medications for older adults increased from 2 to 4 . At the same time, the proportion of patients taking $\geq 5$ medications increased from $13 \%$ to $39 \% .{ }^{1}$ In the oncologic setting, the proportion of older adults with cancer taking $\geq 5$ medications is $>80 \% .^{2}$ Moreover, some medications fall under the umbrella of potentially inappropriate medications (PIMs), which are those flagged as having more risk than benefit for older adults. In 2018, 7.3 billion doses of PIMs were dispensed in the United States for older adults. ${ }^{3}$ As a result, it is quite common for oncologists to encounter older adults with cancer who are taking numerous medications. Furthermore, it is extremely likely that due to additionally prescribed cancer-related medications, this medication list becomes even longer.

Polypharmacy is a complex issue that can often lead to nonadherence, adverse drug reactions, drug-drug interactions, increased emergency room visits, hospitalizations, and nursing home admissions. ${ }^{4}$ Although management of a patient's polypharmacy is not the oncologist's primary responsibility, review of the medication list by the oncology team is crucial. There might be drug-drug interactions between a patient's cancer treatment and non-cancer-related medications, which can impact both the efficacy and toxicity of various cancer treatments. Moreover, reviewing the medication list allows the oncologist to modify potentially toxic cancer treatment (eg, not administering neurotoxic chemotherapy when the patient is on high doses of other medications to manage severe diabetic neuropathy).

The study by Mohamed et al, ${ }^{5}$ elsewhere in this issue, advances the discussion on the importance of a comprehensive medication evaluation, including over-thecounter and complementary medication use, among older adults with cancer prior to starting chemotherapy in order to identify polypharmacy and PIMs. This study also determined the optimal cutoff for polypharmacy in older adults newly diagnosed with cancer. Among 439 patients with cancer aged $\geq 70$ years, taking $\geq 8$ medications had the strongest association with physical function impairments, such as activities of daily living. In this study, investigators did not find a meaningful relationship between taking $\geq 5$ medications and any physical function measures, even though taking $\geq 5$ medications is the most commonly used definition of polypharmacy. This finding suggests that a simple check of a patient's medication list and the number of medications that an older adult with cancer is taking can identify potential functional impairment.

The study also highlights the high prevalence of PIMs (62\%) in older patients with cancer, which was determined using the American Geriatrics Society Beers Criteria and was also found to be correlated with physical functional impairments. Mohamed et al ${ }^{5}$ completed an in-depth PIM assessment by applying all 4 parts of the Beers Criteria, rather than just the first part of the criteria, which is more often used. Classes of commonly found PIMs were proton pump inhibitors, benzodiazepines, nonsteroidal anti-inflammatory drugs, and first-generational antihistamines. The Beers Criteria for screening PIM use in older adults has been widely used as a guide in analyzing prescribing trends in older adults and applies to the general hospital and long-term care facility settings. Using the criteria to prevent polypharmacy and PIMs is straightforward and effective.

See page 267 for related article.

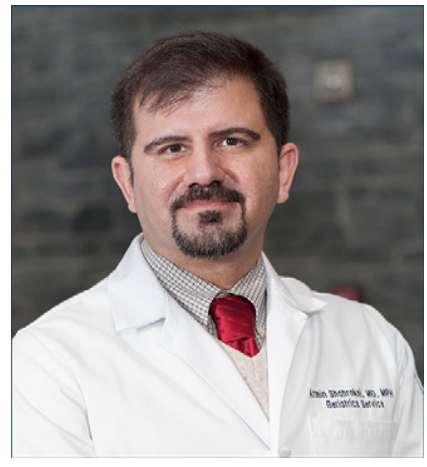

ARMIN SHAHROKNI, MD, MPH

Armin Shahrokni, MD, MPH, is a Geriatrician and Colorectal Oncologist at Memorial Sloan Kettering Cancer Center (MSKCC) for the past 7 years. His primary area of research interest is on outcomes of older adults with cancer following different treatment modalities. He has led the efforts at MSKCC to develop and implement electronic Rapid Fitness Assessment, a web-based geriatric assessment, as a routine care.

doi: $10.6004 /$ jnccn.2021.7025

The ideas and viewpoints expressed in this commentary are those of the author and do not necessarily represent any policy, position, or program of NCCN. 


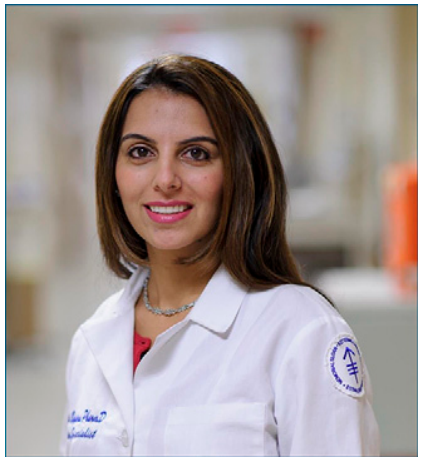

MANPREET K. BOPARAI, PharmD, CGP, BCACP

Manpreet K. Boparai, PharmD, CGP, $\mathrm{BCACP}$, has been a Geriatric-Oncology Clinical Pharmacist at Memorial Sloan Kettering Cancer Center for more than 10 years. Her primary areas of interest are medication management and polypharmacy in older adults with cancer. She is committed to advancing healthcare literacy in her patients as well as the community. She has also held faculty positions at the university level.
Mohamed et al ${ }^{5}$ also discuss the pharmacist's role in a geriatric oncology clinic to assess medication appropriateness, polypharmacy, PIMs, and deprescribing in order to optimize medication use in this population. In other clinic settings, pharmacists have been effectively used to identify polypharmacy and PIMs. ${ }^{6}$

Still, there are significant questions that warrant further study. The trajectory of polypharmacy and functional impairment needs to be studied in a longitudinal study, because there are medications that can accelerate or slow the functional decline. Furthermore, ideally a randomized study evaluating the benefit of deprescribing or other interventions aimed at polypharmacy and PIMs is highly needed.

In short, the next time you have an appointment with an older adult with cancer in your clinic, ask them to bring their pill bottles. The crystal ball of pill bottles will tell you more about the patient's functional impairment and how you need to adjust your treatment plan accordingly.

Disclosures: The authors have reported no relevant financial disclosures.

Funding: The commentary was supported in part by the Beatriz and Samuel Seaver Foundation, the Memorial Sloan Kettering Cancer and Aging Program, and the NIH/NCl Cancer Center Support Grant P30 CA008748.

Correspondence: Armin Shahrokni, MD, MPH, Geriatrics Service, Department of Medicine, Memorial Sloan Kettering Cancer Center, Box 205, 1275 York Avenue, New York, NY 10065 Email: shahroka@mskcc.org

\section{References}

1. Charlesworth CJ, Smit E, Lee DSH, et al Polypharmacy among adults aged 65 years and older in the United States: 1988-2010. J Gerontol A Biol Sci Med Sci 2015;70:989-995.

2. Prithviraj GK, Koroukian S, Margevicius S, et al Patient characteristics associated with polypharmacy and inappropriate prescribing of medications among older adults with cancer. J Geriatr Oncol 2012;3:228-237.

3. Fralick M, Bartsch E, Ritchie CS, et al Estimating the use of potentially inappropriate medications among older adults in the United States. J Am Geriatr Soc 2020;68:2927-2930.

4. Turner JP, Shakib S, Singhal N, et al Prevalence and factors associated with polypharmacy in older people with cancer. Support Care Cancer 2014;22:1727-1734.

5. Mohamed MR, Ramsdale E, Loh KP, et al. Association of polypharmacy and potentially inappropriate medications with physical functional impairments in older adults with cancer. J Natl Compr Canc Netw $2021 ; 19: 267-274$.

6. Nightingale G, Hajjar W, Swartz K, et al Evaluation of a pharmacist-led medication assessment used to identify prevalence of and associations with polypharmacy and potentially inappropriate medication use among ambulatory senior adults with cancer. J Clin Oncol 2015;33:1453-1459. 